\title{
A Spectroscopic and Photometric Study of the Shell Star EW Lac
}

\author{
Huilai $\mathrm{Cao}^{1,2,4}$, Zhongwei $\mathrm{Li}^{3}$, Lin Huang ${ }^{4}$ and Yulian $\mathrm{Guo}^{4}$ \\ ${ }^{1}$ National Astronomical Observatories, Chinese Academy of Sciences, \\ Beijing 100012, China \\ ${ }^{2}$ Beijing Astrophysics Center, Peking University, Beijing 100871, China \\ ${ }^{3}$ Astronomy Department, Beijing Normal University, Beijing 100875, \\ China \\ ${ }^{4}$ Beijing Astronomical Observatory, Beijing 100012, China
}

\begin{abstract}
We report on combined spectroscopic and photometric observations of EW Lac during its outburst of 1983 . At this time the star's $\mathrm{H} \alpha$ and $\mathrm{H} \beta$ lines showed strong doubled-peaked emission profiles which were variable with time. $U B V$ photometry exhibited a long-term and frequent erratic short-term variations. The amplitude of $U, B$ and $V$ variations reached about 0.3 magnitude on October 15, 1983. Both $\mathrm{H} \beta$ emission and the structure of the shell absorption lines of the star also displayed anomalous behavior in 1983. It is evident that this star was in a strong active phase at that time.
\end{abstract}

\section{Introduction}

EW Lac is a typical Be shell star which has been observed for over a century. Although spectra first obtained in 1887, 1904 and 1905 showed no emission features, Frost (1919) later found it to be a B emission line star. Thereafter, the emission and the shell absorption components in Balmer lines and some metallic lines periodically appeared and disappeared. For some fifty years after the mid-1920s, this star exhibited no remarkable changes in spectroscopic and photometric observations. Then, by the end of the 1970s, several authors reported successively that EW Lac showed remarkable asymmetries in Balmer and metallic lines, noticeable changes in the emission profile and radial velocity (Hirata and Kogure 1979). Hubert et al. (1987) presented a detail study on the behavior before and during an active shell phase based on observations during 1960-1984. Kogure and Suzuki (1984) reported long-term V/R variations and the progressive appearance of a $V / R$ asymmetry from higher to lower Balmer lines. In addition to long-term variations, rapid changes of Balmer emission strength and profile shape on time scales of months to less than a day have been reported by some authors (Slettebak 1978 and Ballereau et al. 1987). More recently, Floquet et al. (1992) obtained multiperiodic spectroscopic variations and found moving-bumps in He I $\lambda 6678 \AA$.

The photometric behavior of EW Lac has also attracted several investigators. Walker (1953) first detected short-term light variability with a period of about 


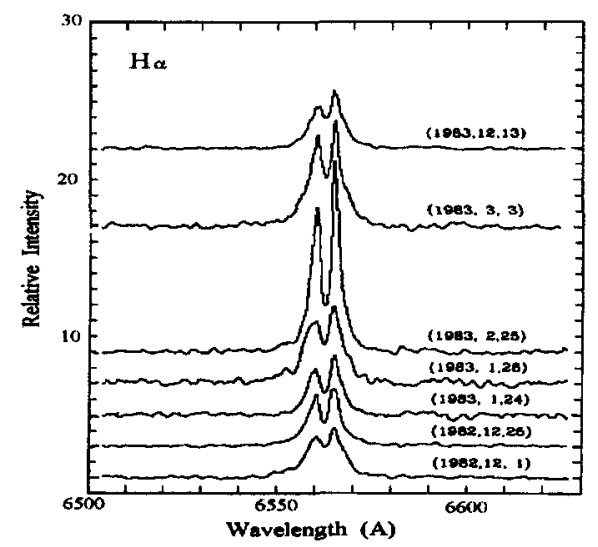

Figure 1. The variations of $\mathrm{H} \alpha$ line profiles obtained at Xinglong Observatory from 1982.12.01-1983.12.13

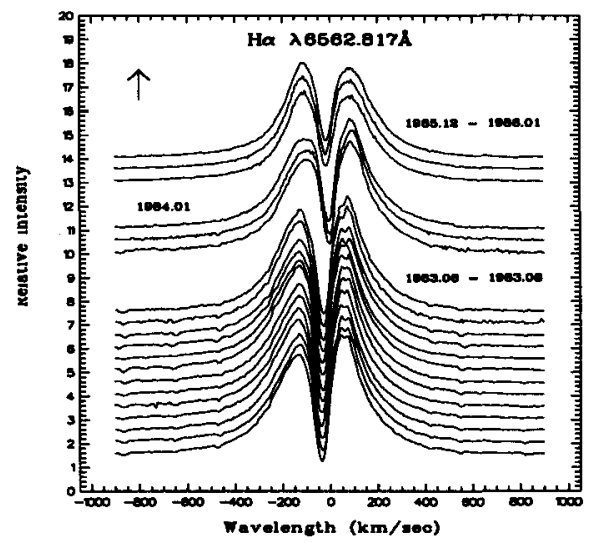

Figure 2. $\mathrm{H} \alpha$ spectroscopy of the Be star EW Lac observed at McDonald Observatory from 1983.5-1986.0.

0.8 day and a light amplitude up to 0.2 magnitude. Similar rapid changes also were discussed by Lester (1975) and Pavlovski (1987), but they have no periodicity and the amplitude also was variable in different observational seasons. Photometric light curves from several international campaigns also showed longterm variations (Harmanec et al., 1980, Kogure 1984).

\section{Observations and data processing}

Spectroscopic and photometric data presented in this paper were obtained at the Xinglong Station of Beijing Observatory except for one spectrum taken at McDonald observatory. The data covered a time span 1982 November - 1996 December for spectroscopy and 1983 October-1990 September for photometry.

Spectroscopy: Kodak 103a-F plates were obtained used the grating spectrograph attached to the $60 / 90 \mathrm{~cm}$ Schmidt telescope during 1982 November1990 January. The spectral dispersion was $50 \AA \mathrm{mm}^{-1}$ and $86 \AA \mathrm{mm}^{-1}$, and the spectral range is $\lambda 3650-6700 \AA$. Spectrograms were digitized on the PDS microdensitometer and further reduced using Starlink software. Other spectra were taken with the coudé spectrograph used Reticon detector at the $2.1 \mathrm{~m}$ telescope of McDonald Observatory during 1983.5-1986.0 (Fig. 2) and CCD spectra at Xinglong Observatory in late 1991.0 and 1991.9. To characterize the star's envelope activity, we measured the radial velocities of the stronger shell lines on selected high-quality spectrograms. The errors in the velocity determination is $\pm 3.9 \mathrm{~km} \mathrm{sec}^{-1}$ for the photographic material.

Photometry: The photometric observations has been made on the $60-\mathrm{cm}$ reflector in the standard $U B V$ system, it was observed together with a number of other program Be stars for which each Be star included a group of comparison, check and red standards stars. For EW Lac itself the comparison, check and red standards stars were 10 Lac, 7 And and 4 And, respectively. 


\section{Spectroscopic results}

Emission lines: In our spectra, the $\mathrm{H} \alpha$ and $\mathrm{H} \beta$ emission lines of the star are strong and double emission peaks. The wings of $\mathrm{H} \gamma$ and $\mathrm{H} \delta$ emission, $\mathrm{Ca}$ II-K and some metallic lines seem to be affected by emission because the lines became very weak at times. A series of the $\mathrm{H} \alpha$ profiles normalized to the continuum is shown in Fig. 1. The $\mathrm{H} \alpha$ emission was strong in 1983-1985 and weakened thereafter, all the time exhibiting fluctuations. The emission equivalent width attained a maximum value of $78 \AA$ in 1983 February and decreased slowly after 1990 to a minimum value of $20 \AA$ in 1993 January. We also observed an outburst in 1983, during the course of which the $\mathrm{H} \alpha$ emission increased suddenly and then decreased gradually. During this time variations of the equivalent width correlated with either the violet and red emission peaks in the sense that the V/R ratio decreased slightly with increased $\mathrm{H} \alpha$ emission strengthening, and vice versa. In addition, we note that before 1985.0 the ratio $V / R<1$ but increased to above unity over the next five years. Occasionally, both $\mathrm{H} \alpha$ and $\mathrm{H} \beta$ changed erratically from $>1$ to $<1$ over several hours.

Shell absorption lines: The shell absorption lines in Balmer series (from $\mathrm{H} \gamma$ to $\mathrm{H} 9$ ) were strong in the EW Lac spectrum. Sharp absorption cores were visible up to $\mathrm{H} 20$. At the end 1983 the Balmer shell absorption lines of the star underwent a remarkable variation. At this time a single sharp, deep absorption core appeared on December 16, 1983. By two nights later this profile had changed to a double absorption core. After several day more days, a central reversal of $\mathrm{H} \alpha$ developed broad, shallow double cores. In a spectrum taken on December 27, 1983 the red component of the two Balmer absorption cores appeared much weaker than the violet component. Two days later, these lines again showed single sharp, deep cores like the profile observed on December 16, 1983. The absorptions of Ca II-K and some metallic line (Fe II $\lambda 4923$ and 5018 $\AA$ ) profiles might be have double components too, but our data quality for this night was too low for us to be sure.

Radial velocities of the shell lines: Many shell absorption line of EW Lac are strong and sharp in our spectra. Thus, we could measure reliable line displacements. To determine radial velocities, we selected high-quality spectrograms and measured positions of the strong lines $\mathrm{H}$ and $\mathrm{K}$ shell lines. The variations of radial velocity along the Balmer series are plotted in Fig. 3 for some representative spectra. Our results are as follows:

(1) no Balmer progression could be detected during our observing seasons,

(2) the radial velocities changed erratically with time,

(3) when the Balmer shell absorption lines showed double structure in the end of 1983 , the velocities of the $R$ component remain nearly the same as these of the single absorption lines and the velocities of the $\mathrm{V}$ component become more negative and change with time,

(4) the radial velocity measures for the Ca II-K line were consistent with those of the Balmer lines on a given spectrogram.

\section{Photometric results}

The photoelectric results of this star are shown in Figure 4. We show mean nightly values of $U, B, V, B-V$ and $U-B$ magnitudes during 1983 October- 

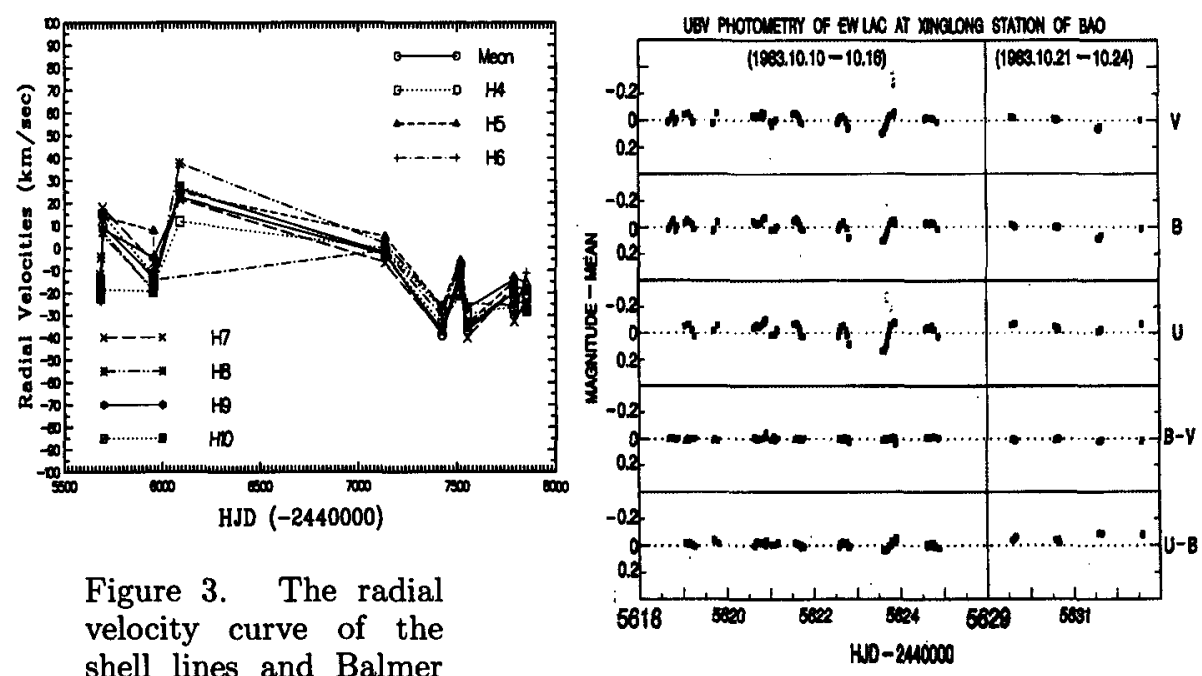

Figure 3. The radial velocity curve of the shell lines and Balmer line series during 1984.0-1989.9 (HJD244 $5685-7854)$

Figure 4. Photometric observations during Oct. 10-24, 1983

1993 September versus Julian Date. We found that the brightness of EW Lac displayed significant variations with time and that these variations occurred together in all three bandpasses. The star became faintest at the end of both 1986 and 1990, and increased gradually subsequent to these times.

The photometry of EW Lac also exhibited erratic short-term variations. The rapid variations in 1983 were most marked. The amplitude of the $U, B$ and $V$ reached about 0.3 magnitude in the night of October 15, 1983. Both the $\mathrm{H} \beta$ emission and the structure the shell absorption lines of the star displayed anomalous behavior in 1983. It is evident from these observations that this star was in a particularly active phase at that time.

\section{References}

Ballereau, D., Alvarez, M. et al. 1987, Rev. Mex. Astr. Astrof. 15, 29

Floquet, A., Hubert, A.M. et al. 1992, A\&A 264, 177

Frost, E.B. 1919, ApJ 49, 61

Hamanec, P., Horn, J., Koubsky, P. et al. 1980, Bull. Astr. Inst. Cz. 31, 144

Hirata, R., and Kogure, T. 1979, Inf. Bull. Var. Stars No. 1575

Hubert, A M., Floquet, M. et al. 1987, A\&A 70, 443

Kogure, T., and Suzuki, M. 1984, PASJ 36, 191

Lester, D.F. 1975, PASP 87, 177

Pavlovski, K. 1987, ApSS 134, 317

Slettebak, A., Reynolds, R.C. 1978, ApJS 38. 205

Walker, M.F. 1953, ApJ 118, 481 